G62(P)

\section{NEUROCYSTICERCOSIS IN A BRITISH TEENAGER PRESENTING AS A SPACE OCCUPYING LESION CAUSING FOCAL SENSORY-MOTOR SEIZURES IN LONDON}

${ }^{1} \mathrm{~S}$ Tilib-Shamoun, ${ }^{2} \mathrm{~B}$ Bodi, ${ }^{3} \mathrm{G}$ Godbole, ${ }^{4} \mathrm{~A}$ Siddiqui, ${ }^{5} \mathrm{~B}$ Zebian, ${ }^{1} \mathrm{~V}$ Ramesh. ${ }^{1}$ Department of Paediatric Neurology, King's College Hospital, London, UK; ${ }^{2}$ Department of Clinical Neuropathology, King's College Hospital, London, UK; ${ }^{3}$ Department of Clinical Parasitology, Hospital for Tropical Diseases, London, UK; ${ }^{4}$ Department of Neuroradiology, King's College Hospital, London, UK; ${ }^{5}$ Department of Neurosurgery, King's College Hospital, London, UK

\subsection{6/archdischild-2020-rcpch.48}

Background Neurocysticercosis is a helminthic infestation of the brain by the encysted larval form of the human tape worm Taenia Solium. It is a common cause of focal epilepsy in countries with inadequate sanitation following oro-faecal transmission and ingestion of food contaminated with TaeniaSolium eggs. The larvae can lodge in different parts of the human body including the brain parenchyma resulting invariable neurological clinical manifestations depending on the affected site. Onset of symptoms depends on the host immune response and on parasite defence mechanism. Over 90\% patients present with focal seizures. Localised oedema surrounding the lesion can result in headaches from raised intracranial pressure, obstructive hydrocephalus. Other forms include spinal cysticercosis and ophthalmic cysticercosis.

The diagnosis is usually made on clinical grounds, confirmed by imaging and immunodiagnostic tests.

Case Report We report a previously well 13 year old girl who presented with a single episode of generalised tonic clonic seizure. She gave a 4 month history of altered sensation in her right upper limb and 1 month history of focal sensorimotor seizures. Examination showed subtle weakness of the right side of her face and upper arm.

MRI brain scan showed a well defined left parietal lesion in the grey white matter junction with surrounding oedema and was referred to neurosurgery with suspicion of tumour. On review the images were suggestive of atypical infection Cysticercosis or Tuberculoma. She was given Dexamethasone and Levetiracetam with good effect.

At surgery she had a well encapsulated cystic lesion that was excised. Histopathology showed chronic cerebral lesion with parasitic cyst. Microscopy confirmed presence of a tapeworm larva. Following surgery she was started on Albendazole.

Further history revealed regular camping trips at farms and woodland areas. She also swam in ponds and rivers. She did not report tic bites. Her diet included pork. She had been abroad.

Conclusions Unifocal Neurocysticercosis in non-endemic countries is in the list space occupying lesions mistaken for a brain tumour. It presents with focal epilepsy and its diagnosis can be challenging.

\section{G63(P) TREATMENT PATTERNS IN SPINAL MUSCULAR ATROPHY: INTERVIEWS WITH HEALTHCARE PROFESSIONALS IN THE UNITED KINGDOM}

${ }^{1} \mathrm{O}$ Dabbous, ${ }^{2} \mathrm{~N}$ Germain, ${ }^{1} \mathrm{~B}$ Maru, ${ }^{2} \mathrm{~S}$ Aballéa, ${ }^{2} \mathrm{M}$ Desroches, ${ }^{2} \mathrm{M}$ Toumi, ${ }^{3} \mathrm{~J}$ Loukes ${ }^{4} \mathrm{R}$ Arjunji. ${ }^{1}$ Global Health Economics and Outcomes Research and Real World Evidence, AveXis, Inc., Bannockburn, Illinois, USA; ${ }^{2}$ Health Economics and Outcomes Research, Creativ-Ceutical, Paris, France; ${ }^{3}$ UK Medical Affairs, AveXis, Inc., Bannockburn, Illinois, USA; ${ }^{4}$ Health Economics and Outcomes Research and Real World Evidence, AveXis, Inc., Bannockburn, Illinois, USA

10.1136/archdischild-2020-rcpch.49
Aims Spinal muscular atrophy (SMA) is a rare genetic disorder characterized by weakness and atrophy in skeletal muscles. Excluding the prenatal onset form (type 0), SMA is classified into 4 phenotypes based on severity, with type 1 being the most severe. The recent advent of the first disease-modifying therapies has dramatically improved the outlook for patients with SMA, but little is known about current treatment patterns. The objective of this study was to collect data on standard of care treatment patterns in SMA types 1, 2, and 3 in the UK prior to the availability of disease-modifying therapies through health care professional (HCP) interviews.

Methods HCPs who had seen at least two patients with SMA in the past 12 months were recruited through contacts in clinical centers in the UK. Informed consent was obtained from all respondents with subsequent telephone interviews.

Results Interviews were conducted with $16 \mathrm{HCPs}$ from eight UK regions: nine neurologists, three pulmonologists, two physiotherapists, one nurse, and one health visitor. Participants reported that paths to diagnosis depend on symptom timing and HCPs involved. Time to diagnosis was estimated at up to 3 months for SMA type 1, and up to 12 months for SMA types 2 and 3. HCPs agreed on key approaches to management and care coordination. Patients' geographic proximity to a hospital often determines who coordinates care. Although the concept of segmenting SMA disease progression into defined, discrete phases was controversial, there was broad concurrence on key parameters of ventilation, nutrition, and mobility. Many HCPs are involved in patient care, with community support being essential; however, HCPs were not able to report on palliative care and personal social services. The challenges faced by HCPs in SMA are numerous and include a lack of resources and a lack of effective treatment options. Conclusion HCP reports underline the complexity of treatment coordination for SMA between hospitals and communities. Management is focused on ventilation, feeding, and mobility, with the involvement of a large number of HCPs. A comprehensive SMA care model is needed.

\section{G64(P) ABSTRACT WITHDRAWN}

\section{G65(P) ABSTRACT WITHDRAWN}

\section{G66(P) ABSTRACT WITHDRAWN}

\section{G67(P) A RARE DIAGNOSIS OF LAMB-SHAFFER SYNDROME IN TWINS WITH KLINEFELTER'S}

S Rhead, N Weerapperuma, C Robertson. Community Paediatric Department, Oxford University Hospitals, Oxford, UK

\subsection{6/archdischild-2020-rcpch.50}

A set of twin brothers with Klinefelter's syndrome were seen in community paediatric clinic following concern about severe learning disabilities requiring special educational needs school. 
As Klinefelter's is not commonly associated with severe learning disabilities, they were enrolled in the 100,000 genome project. Whole exome sequencing revealed a mutation involving the SOX5 gene on chromosome 12p12, which is associated with Lamb-Shaffer syndrome. Lamb-Shaffer syndrome is a neurodevelopmental disorder characterised by global developmental delay, learning disability, poor expressive speech and mild dysmorphic facial features (Nesbitt et al 2015). Less than 100 cases have been reported since the condition was first described in 2012.

This new genetic diagnosis has enabled the health care professionals and the parents to understand more about the clinical features, including what surveillance and investigations the patients may require based upon other reported cases with the same mutation.

For parents and patients, having a named diagnosis allows them to connect via support groups with other families who have been diagnosed with the same condition. Historically the 'diagnostic odyssey' in a child with a rare disease could continue for many years, with serial testing of different genes, often with no answers found for patients or their parents. Learning disability is genetically heterogeneous and has previously presented challenges for molecular diagnoses using traditional genetic testing methods. Next generation sequencing has made it possible to sequence millions of fragments of DNA simultaneously, enabling the sequencing of whole patient exomes as part of the 100,000 genome project. These patients are two of many new genetic diagnoses that are a result of the genome project, which are highlighting the utility of exome sequencing in clinically and genetically heterogeneous conditions such as learning disability.

In this unusual case these twins have two different genetic underlying conditions, which demonstrates the importance of investigating further possible causes when previous diagnoses do not explain the evolving clinical phenotype.

\section{G68(P) ABSTRACT WITHDRAWN}

\section{G69(P) PARENT-DELIVERED STRATEGIES TO IMPROVE EATING, DRINKING AND SWALLOWING DIFFICULTIES OF YOUNG CHILDREN WITH NEURODISABILITY: CURRENT PRACTICE AND FUTURE RESEARCH}

1,2,3 Parr 1,2 Lennington, ${ }^{1} \mathrm{H}$ Taylor, ${ }^{4} \mathrm{C}$ Morris, ${ }^{1} \mathrm{H}$ McConachie, 1,5,6] Cadwoan, ${ }^{7}$ D Sellers, 1,2 FEEDS co-investigators. ${ }^{1}$ Population and Health Sciences Institute, Newcastle University, Newcastle upon Tyne, UK; ${ }^{2}$ Great North Children's Hospital, Newcastle upon Tyne Hospitals NHS FT, Newcastle upon Tyne, UK; ${ }^{3}$ Complex Neurodevelopmental Disorders Service, Cumbria, Northumberland, Tyne and Wear NHS FT, Newcastle upon Tyne, UK; ${ }^{4}$ Peninsula Cerebra Research Unit (PenCRU), University of Exeter, Exeter, UK; ${ }^{5}$ Evelina London Children's Hospital, Guys and St Thomas' NHS FT, King's Health Partners, London, UK; ${ }^{6}$ Kings College London, London, UK; ${ }^{7}$ Sussex Community NHS FT, Chailey Clinical Services, Sussex, UK

\subsection{6/archdischild-2020-rcpch.51}

Aims Eating, drinking and swallowing difficulties (EDSD) are common in young children with neurodisability. EDSD can be caused by physical and non-physical difficulties leading to inadequate calorie intake, affecting a child and parents everyday life. Guidance on how to address these difficulties is lacking. We undertook a study to answer the question: What interventions, which could be delivered at home by parents, are available to improve eating in young children with neurodisability and are suitable for investigation in pragmatic trials?

Methods We undertook: 1. Updates of three systematic reviews, and a mapping review of the research evidence for interventions and outcome measures. 2. Two national parent and professional surveys: one about current professional practice and what is received by parents, and a Delphi survey to identify the most important outcomes. 3. Focus groups with parents, professionals and young people to discuss current practice and workshops identify future directions and research.

Results 951 parents and professionals participated. The limitations of the research evidence regarding interventions and tools to measure outcomes was identified. The survey showed a wide range of interventions are used in NHS practice parents and professionals reported variability in provision. Nonetheless, parents and professionals considered many interventions were important, and led to change in EDSD. They identified 19 interventions as important to deliver when needed, and 10 outcomes that were most important to measure (including nutrition, growth, and health/safety); young people agreed these were important outcomes. Parents and professionals were positive about a proposed 'toolkit' of interventions that through shared decision making, could be offered by health professionals.

Conclusions The challenges for future clinical trials of a toolkit of interventions were evident (for example, the range of interventions that children in a comparator group might receive). Parents and professionals thought that implementation of the toolkit as part of usual practice would be a positive step. However, this would require the toolkit to be operationalised through further development - evaluation of its effectiveness could then be undertaken.

\section{G70(P) NITROUS OXIDE ABUSE RESULTING IN SUB-ACUTE DEGENERATION OF THE SPINAL CORD - A CASE REPORT}

RL Horner, A Shields, R Ramiah. Paediatrics, University Hospital Coventry and Warwickshire, Coventry, UK

\subsection{6/archdischild-2020-rcpch.52}

A case report of a 15 year old boy presenting with a 3 week history of progressive ataxia and sensory loss. Examination revealed complete bilateral sensory loss from toes to mid-thigh and sensory loss in both little fingers. He admitted to heavy inhalational abuse of nitrous oxide and daily use of cannabis. No other medical problems and no family history of note. Blood tests revealed a normal total vitamin B12 level (247 ng/L), but raised methylmalonic acid (500 $\mathrm{nmol} / \mathrm{L}$ ) and reduced active vitamin B12 (30 pmol/L), this was indicative of an active vitamin B12 deficiency. An MRI demonstrated high signal changes in the posterior cord extending from $\mathrm{C} 1$ to the lower thoracic cord which was typical of sub-acute degeneration of the spinal cord. Vitamin B12 deficiency is known to cause sub-acute combined degeneration of the spinal cord. He was treated with daily vitamin B12 injections and showed some clinical recovery whilst an inpatient. 